\title{
Contribution of PRS3, RPB4 and ZWF1 to the resistance of industrial Saccharomyces cerevisiae CCUG53310 and PE-2 strains to lignocellulosic hydrolysate-derived inhibitors
}

\author{
Joana T. Cunha, Tatiana Q. Aguiar, Aloia Romaní, Carla Oliveira, Lucília Domingues* \\ CEB - Centre of Biological Engineering, University of Minho, 4710-057 Braga, Portugal
}

\section{H I G H L I G H T S}

- Expression analyses revealed PRS3, RPB4 and ZWF1 roles in adaptation to inhibitors.

- PRS3, RPB4 and ZWF1 were overexpressed in two industrial S. cerevisiae strains.

- Fermentations were conducted in Eucalyptus globulus wood and corn cob hydrolysates.

- PRS3 overexpression revealed to be advantageous for lignocellulosic fermentation.

- PRS3, RPB4 and ZWF1 overexpression results differed depending on strain/hydrolysate.

\section{A R T I C L E I N F O}

\section{Article history:}

Received 31 March 2015

Received in revised form 30 April 2015

Accepted 2 May 2015

Available online 7 May 2015

\section{Keywords:}

Second generation bioethanol

Lignocellulose-derived inhibitors

Industrial Saccharomyces cerevisiae

PRS3, RPB4 and ZWF1

Expression and overexpression analysis

\begin{abstract}
A B S T R A C T
$P R S 3, R P B 4$ and ZWF1 were previously identified as key genes for yeast tolerance to lignocellulose-derived inhibitors. To better understand their contribution to yeast resistance to the multiple stresses occurring during lignocellulosic hydrolysate fermentations, we overexpressed these genes in two industrial Saccharomyces cerevisiae strains, CCUG53310 and PE-2, and evaluated their impact on the fermentation of Eucalyptus globulus wood and corn cob hydrolysates. PRS3 overexpression improved the fermentation rate (up to $32 \%$ ) and productivity (up to $48 \%$ ) in different hydrolysates. ZWF1 and RPB4 overexpression did not improve the fermentation performance, but their increased expression in the presence of acetic acid, furfural and hydroxymethylfurfural was found to contribute to yeast adaptation to these inhibitors. This study expands our understanding about the molecular mechanisms involved in industrial yeast tolerance to the stresses occurring during lignocellulosic bioethanol production and highlights the importance of selecting appropriate strain backgrounds/hydrolysates combinations when addressing further improvement of these processes.
\end{abstract}

(c) 2015 Elsevier Ltd. All rights reserved.

\section{Introduction}

One of the major challenges faced in the production of bioethanol and other value-added products from lignocellulosic biomass is the generation of a wide range of compounds during the pre-treatment and hydrolysis process that affect the physiology and metabolism of microorganisms, decreasing their viability and productivity (Chandel et al., 2013; Parawira and Tekere, 2011). Therefore, the commercial success of lignocellulosic biomass conversion necessarily depends on the development of microorganisms able to cope with these inhibitors while simultaneously

\footnotetext{
* Corresponding author. Tel.: +351253 604400; fax: +351253604429.

E-mail address: luciliad@deb.uminho.pt (L. Domingues).
}

produce satisfactory amounts of the desired product (Chandel et al., 2013).

The inherent ability of yeast cells to withstand and detoxify the main inhibitory compounds present in lignocellulosic hydrolysates (acetic acid, furfural and hydroxymethylfurfural (HMF)) is variable among strains and determinant for efficient lignocellulosic bioethanol production (Modig et al., 2008; Pereira et al., 2014a). Depending on their genetic background, yeast cells activate distinct gene expression programs to help them counteract the negative impact of these inhibitors on their metabolism (Liu et al., 2009). Strains isolated from harsh industrial environments, such as Saccharomyces cerevisiae CCUG53310 (Purwadi et al., 2007) and PE-2 (Basso et al., 2008) (isolated from second and first generation bioethanol plants, respectively), have been shown to be more efficient in detoxifying and fermenting lignocellulosic hydrolysates 
than other industrial and laboratory background strains (Pereira et al., 2014a; Westman et al., 2012). However, the molecular characterization of these strains under relevant process conditions is limited, which hampers the understanding of the molecular mechanisms underlying their tolerance to inhibitory hydrolysates and the further improvement of their resistance to these compounds.

Results obtained from chemogenomic (Alriksson et al., 2010; Gorsich et al., 2006; Mira et al., 2010; Pereira et al., 2011, 2014b) and transcriptomic (Bajwa et al., 2013; Liu et al., 2009; Ma and Liu, 2010) analyses have been helpful in identifying the genetic determinants of yeast tolerance to lignocellulose-derived inhibitors. Overexpression of some target genes identified through these approaches in laboratory background strains has already been shown to be associated with improved growth, fermentation rate and/or ethanol production in the presence of these inhibitors (Alriksson et al., 2010; Gorsich et al., 2006; Park et al., 2011; Petersson et al., 2006). Based on chemogenomic analyses, we have previously identified PRS3 and RPB4 as key genes necessary for yeast growth and maximal fermentation rate in wheat straw hydrolysate (Pereira et al., 2011, 2014b), but the outcome of their overexpression in $S$. cerevisiae lignocellulosic-based fermentations has not yet been assessed. ZWF1, which had been previously shown to confer resistance to furfural and HMF (Gorsich et al., 2006; Park et al., 2011), was also found to be important for maximal yeast resistance to wheat straw hydrolysate (Pereira et al., 2014b).

Genetic strategies addressing strain tolerance improvement to lignocellulose-derived inhibitors have been developed mainly through gene overexpression in laboratory background strains and tested using synthetic hydrolysates (Gorsich et al., 2006; Park et al., 2011). Nevertheless, strains with robust genetic backgrounds have been shown to already display enhanced background expression of several genes involved in the detoxification of some of these inhibitors (Liu et al., 2009), and therefore their extra expression may result in different outcomes from those reported for laboratorial background strains. Moreover, the inhibitory load of lignocellulosic hydrolysates, which varies depending on the raw material and operational conditions of pretreatment (Chandel et al., 2013), has been shown to differentially influence the outcome of genetic manipulations (Alriksson et al., 2010; Park et al., 2011; Wallace-Salinas et al., 2014), highlighting the importance of evaluating their effect under process-like conditions.

In this work, we aimed at evaluating the contribution of PRS3, RPB4 and ZWF1 for the resistance of the industrial $S$. cerevisiae CCUG53310 and PE-2 strains to lignocellulosic hydrolysatederived inhibitors, as a means to elucidate their role on yeast response to the multiple stresses occurring during lignocellulosic ethanol production and to better understand the molecular mechanisms underlying the robustness of these strains. For that, we analysed the expression profiles of these genes in fermentations with and without acetic acid, furfural and HMF, and evaluated the effect of their overexpression on the fermentation performance of both strains in corn cob and Eucalyptus globulus wood (EGW) hydrolysates, which contain different inhibitory loads.

\section{Methods}

\subsection{Strains}

Two industrial S. cerevisiae strains were used in this work: CCUG53310, flocculating strain isolated from a Swedish second generation bioethanol plant (Purwadi et al., 2007); and PE-2, isolated from a Brazilian first generation bioethanol plant (Basso et al., 2008). Overexpressing strains (Table 1 ) were generated using CCUG53310 and PE-2 as parental strains, as described below. S. cerevisiae CEN.PK113-7D was the source of the genomic DNA for
Table 1

Strains, plasmids and primers used in this study. Upper case sequences correspond to sequences complementary to the template. Lower case sequences correspond to additions for restriction sites (underlined).

\begin{tabular}{|c|c|c|}
\hline & Relevant features & Source \\
\hline \multicolumn{3}{|l|}{$\begin{array}{l}\text { S. cerevisiae } \\
\text { strains }\end{array}$} \\
\hline CEN.PK113-7D & MAT $\alpha$, MAL2-8c, SUC2 & $\begin{array}{l}\text { INSA, } \\
\text { France }\end{array}$ \\
\hline CCUG53310 & Flocculation & $\begin{array}{l}\text { (Purwadi } \\
\text { et al., 2007) }\end{array}$ \\
\hline PE-2 & Diploid & $\begin{array}{l}\text { (Basso et al., } \\
\text { 2008) }\end{array}$ \\
\hline CC-Yep & CCUG53310, YEplac195KanMX & This work \\
\hline CC-ZWF1 & CCUG53310, YEpJCZ & This work \\
\hline CC-PRS3 & CCUG53310, YEpJCP & This work \\
\hline CC-RPB4 & CCUG53310, YEpJCR & This work \\
\hline PE-Yep & PE-2, YEplac195KanMX & This work \\
\hline PE-ZWF1 & PE-2, YEpJCZ & This work \\
\hline PE-PRS3 & PE-2, YEPJCP & This work \\
\hline PE-RPB4 & PE-2, YEPJCR & This work \\
\hline \multicolumn{3}{|l|}{ Plasmids } \\
\hline YEplac195 & $\begin{array}{l}\text { URA3 marker; 2-micron origin of } \\
\text { replication }\end{array}$ & $\begin{array}{l}\text { Gietz and } \\
\text { Sugino } \\
(1988)\end{array}$ \\
\hline YEplac195KanMX & $\begin{array}{l}\text { URA3 and KanMX marker; 2-micron origin } \\
\text { of replication }\end{array}$ & This work \\
\hline YEpJCZ & $\begin{array}{l}\text { YEplac195KanMX containing the ZWF1 } \\
\text { gene under the control of its native } \\
\text { promoter }\end{array}$ & This work \\
\hline YEpJCP & $\begin{array}{l}\text { YEplac195KanMX containing the PRS3 } \\
\text { gene under the control of its native } \\
\text { promoter }\end{array}$ & This work \\
\hline YEpJCR & $\begin{array}{l}\text { YEplac195KanMX containing the RPB4 } \\
\text { gene under the control of its native } \\
\text { promoter }\end{array}$ & This work \\
\hline Primers & Sequence $\left(5^{\prime}-3^{\prime}\right)$ & \\
\hline KanMX_FW & ggaattccatatgGAGATCTGTTTAGCTTGCCTC & \\
\hline KanMX_RV & ggaattccatatgGCTCGTTTTCGACACTGG & \\
\hline $\mathrm{Z1}$ & GTAAGGTGTAGTTTTGCACCC & \\
\hline $\mathrm{Z2}$ & AAATTTTTGCAGACATTTTTGATATATAT & \\
\hline P1 & TTATCTTCATCACCGCCATAC & \\
\hline P2 & ACAAGAGAAACTTTTGGGTAAAATG & \\
\hline R1 & GATTGCTCAAATTAGCATGTGAA & \\
\hline R2 & AATCCTGTCCTTTTTCCTGTTAAATAG & \\
\hline qPCRZWF1_FW & CTGGTCTGTCAAATGCTACC & \\
\hline qPCRZWF1_RV & CCAGTAGGGCGTCTCTTAT & \\
\hline qPCRPRS3_FW & GGCTAGGTCTACAGTTAACAAG & \\
\hline qPCRPRS3_RV & GTCССТAACAGATTCTCCAATAG & \\
\hline qPCRRPB4_FW & ACGGGAGGAAATAATAAAGATTTG & \\
\hline qPCRRPB4_RV & GACGGTTTCTTGGTCTCTAAAT & \\
\hline qPCRACT1_FW & GCCGAAAGAATGCAAAAGGA & \\
\hline qPCRACT1_RV & TAGAACCACCAATCCAGACG & \\
\hline
\end{tabular}

genes amplification. Escherichia coli NZY5 $\alpha$ (Nzytech) was used as the recipient for all cloning steps.

\subsection{Plasmids construction and yeast transformation}

Expression plasmids containing the $S$. cerevisiae 2-micron replication origin were generated as follows. The KanMX cassette conferring resistance to geneticin/G418 was amplified from pUG6 (Guldener et al., 1996) with the KanMX primers pair (Table 1) and inserted into the NdeI site of YEplac195 (Gietz and Sugino, 1988), generating plasmid YEplac195KanMX. Based on the annotated sequence for the YNL241C (ZWF1), YHL011C (PRS3) and YJL140W (RPB4) open reading frames (NCBI BioProject Accession number PRJNA52955), their complete coding regions with corresponding native promoter and terminator sequences were amplified by PCR from S. cerevisiae CEN.PK113-7D genomic DNA using the primers pairs Z1/Z2, P1/P2 and R1/R2 (Table 1), respectively. 
The PCR products were cloned into the pGEM-T Easy vector (PROMEGA) and the resulting constructs were digested with SacI/SphI, for isolation of the ZWF1 and RPB4 regions, or with $E C o R I$, for isolation of the PRS3 region. The digested fragments were cloned into the YEplac195KanMX vector previously digested with the corresponding enzymes. The resulting ZWF1, PRS3 and RPB4 expression plasmids were named YEpJCZ, YEpJCP and YEpJCR, respectively. The orientation and sequence of the inserts in the plasmids were confirmed by restriction analyses and sequencing (Eurofins MWG Operon) with the primers pairs Z1/Z2, P1/P2 or $\mathrm{R} 1 / \mathrm{R} 2$ (Table 1). Vectors were introduced into $S$. cerevisiae CCUG53310 and PE-2 using the lithium acetate method (Gietz et al., 1992). The empty vector (YEplac195KanMX) was also transformed into both yeast strains to serve as control in the fermentation assays. Transformants were selected in G418-containing media.

\subsection{Preparation of the EGW, corn cob and synthetic hydrolysates}

Lignocellulosic feedstocks (EGW and corn cob) were collected, milled and stored until its use. EGW and corn cob hydrolysates were prepared following optimized conditions previously described in Pereira et al. (2014a) and Romani et al. (2015), respectively. For this, water and lignocellulosic biomass were mixed at Liquid to Solid Ratio (LSR) equal to $8 \mathrm{~kg} / \mathrm{kg}$ and submitted to hydrothermal treatment in a $3.7 \mathrm{~L}$ stainless steel reactor at 202 and $210^{\circ} \mathrm{C}$ for corn cob and EGW, respectively. The hardness of treatment (expressed as "severity"; $S_{0}$ ) is defined as follow:

$S_{0}=\log R_{0}=\log \int_{0}^{t} \exp \frac{T(t)-T_{\mathrm{REF}}}{\omega} d t$

where $T(t)$ stands for the time-temperature profile (including heating isothermal period and cooling). Calculations were made assuming the values usually employed in literature for $T_{\mathrm{REF}}$ and $\omega\left(100{ }^{\circ} \mathrm{C}\right.$ and $14.75^{\circ} \mathrm{C}$, respectively) (Lavoie et al., 2010). The value of $S_{0}$ was 3.84 and 4.10 for corn cob and EGW treatments, respectively. The liquid fractions, hydrolysates containing hemicellulose and lignin-derived compounds, were recovered by filtration and analysed by HPLC (Section 2.7). The composition of these EGW and corn cob hydrolysates was according to that reported by Pereira et al. (2014a) and Romani et al. (2015), respectively. The pH of the hydrolysates was adjusted to 4.5 with $10 \mathrm{M}$ sodium hydroxide. The hydrolysates were sterilized by filtration $(0.2 \mu \mathrm{m}$ pore size sterile filters) and supplemented with approximately $100 \mathrm{~g} / \mathrm{L}$ glucose to improve the ethanol titer. Based on the inhibitors concentration of EGW hydrolysate, several synthetic media were prepared to test the susceptibility of the overexpressing strains to the main inhibitors present in real lignocellulosic hydrolysates, which consisted of a minimal medium containing, per liter, $1.7 \mathrm{~g}$ yeast nitrogen base without amino acids and ammonium sulfate, $5 \mathrm{~g}$ L-asparagine, ca. 100 g glucose, and: (1) acetic acid, furfural and HMF, or (2) only furfural. When indicated, real and synthetic hydrolysates were supplemented with $100 \mu \mathrm{g} / \mathrm{mL}$ G418 for plasmid maintenance.

\subsection{Inoculum preparation}

The strains used to inoculate the fermentation media (Table 1) were cultivated at $30^{\circ} \mathrm{C}$ for $20-22 \mathrm{~h}$, with orbital agitation (200 rpm), in YPD medium, containing, per liter, $10 \mathrm{~g}$ yeast extract, $20 \mathrm{~g}$ peptone and $20 \mathrm{~g}$ glucose. When indicated, $100 \mu \mathrm{g} / \mathrm{mL}$ of G418 were added to the media. Cells were recovered by centrifugation $\left(15 \mathrm{~min}, 4000 \mathrm{~g}, 4^{\circ} \mathrm{C}\right.$ ) and pellets were resuspended in ice-cold $0.9 \%(\mathrm{w} / \mathrm{v})$ sodium chloride to obtain $200 \mathrm{mg}$ of fresh yeast $/ \mathrm{mL}$. Before the resuspension, pellets from the CCUG53310 strain and from the CCUG53310-derived transformants were washed twice with $1.5 \%(\mathrm{w} / \mathrm{v})$ sodium chloride, $\mathrm{pH} 3.0$ to deflocculate. The concentrated cell suspensions were used to inoculate $30 \mathrm{~mL}$ of fermentation media, with a cellular concentration of $5 \mathrm{mg}$ of fresh yeast/mL (mimicking the high initial cell densities used at the industrial scale).

\subsection{Fermentation assays}

Fermentations were carried out in $100 \mathrm{~mL}$ Erlenmeyer flasks fitted with perforated rubber stoppers enclosing glycerol-filled air-locks (to permit $\mathrm{CO}_{2}$ exhaustion while avoiding the entrance of air). Prior to inoculation, the media were aerated by stirring with a magnetic bar (length of $3 \mathrm{~cm}$ ) at $>850 \mathrm{rpm}$ for $20 \mathrm{~min}$. Under these conditions, the oxygen concentration in the growth media was higher than $95 \%$ of air saturation. The fermentations were followed by measuring the reduction of mass loss resulting from $\mathrm{CO}_{2}$ production.

$\mathrm{CO}_{2}$ production was mathematically modeled following the equation described by Rodrigues et al. (2006):

$P=\frac{P_{0} P_{\max } e^{P_{r} t}}{P_{\max }-P_{0}+P_{0} e^{P_{r} t}}$

where $t$ is time $(\mathrm{h}), P$ is $\mathrm{CO}_{2}$ concentration $(\mathrm{g} / \mathrm{L}), P_{\max }$ is maximum concentration of $\mathrm{CO}_{2}(\mathrm{~g} / \mathrm{L})$, and $P_{r}$ is the ratio between the initial volumetric rate of $\mathrm{CO}_{2}$ formation $\left(r_{p}\right)$ and the initial $\mathrm{CO}_{2}$ concentration $P_{0}(\mathrm{~g} / \mathrm{L})$, which represents the maximum fermentation rate. The experimental data was fitted to the model and the parameters $P_{0}$, $P_{\max }$, and $P_{r}$ were calculated for each fermentation by nonlinear regression using the least-squares method using commercial software (Solver of Microsoft Excel 2010). The maximum $\mathrm{CO}_{2}$ productivity $\left(\mathrm{Qp}_{\max } ; \mathrm{g} /(\mathrm{Lh})\right)$ was calculated at the time the $\mathrm{CO}_{2}$ concentration reached its maximum. Samples were taken at the end of the fermentations and analysed by HPLC.

\subsection{Gene expression analysis}

For PRS3, RPB4 and ZWF1 expression analyses, YPD medium (control) and YPD medium supplemented with approximately $3.11 \mathrm{~g} / \mathrm{L}$ acetic acid, $1.66 \mathrm{~g} / \mathrm{L}$ furfural and $0.33 \mathrm{~g} / \mathrm{L} \mathrm{HMF}$ (concentrations similar to those present in the EGW hydrolysate) were used. Fermentations were carried out as indicated in Section 2.5. Samples containing approximately $2 \times 10^{7}$ cells were collected at different phases of the S. cerevisiae CCUG53310 and PE-2 fermentations: late lag-phase (ca. $1 \mathrm{~g} / \mathrm{L}$ of $\mathrm{CO}_{2}$ produced) and initial exponential phase (ca. $5 \mathrm{~g} / \mathrm{L}$ of $\mathrm{CO}_{2}$ produced). The cell pellet of each sample was immediately stored at $-70{ }^{\circ} \mathrm{C}$ after washing with ice-cold $0.9 \%(\mathrm{w} / \mathrm{v})$ sodium chloride, and the supernatant was stored at $-20^{\circ} \mathrm{C}$ for HPLC analysis (Section 2.7).

Total RNA was extracted from frozen cells with the RNeasy Plant Mini Kit (Qiagen) according to manufacturer's instructions and treated with DNase I (Fermentas). The concentration and purity of the total RNA was spectrometrically determined using a NanoDrop 1000TM (Thermo Scientific) and its integrity assessed on $1 \%$ agarose gel by visualization of the $25 \mathrm{~S} / 18 \mathrm{~S}$ rRNA banding pattern after electrophoresis. Single-stranded cDNA was synthesized from $1 \mu \mathrm{g}$ of total RNA with the SuperScript II Reverse Transcriptase (Invitrogen) and Oligo(dT) primers (NZYTech). Oligonucleotides for quantitative PCR (qPCR; primers with prefix qPCR inTable 1) were designed using the IDT PrimerQuest tool followed by a BLAST analysis against the $S$. cerevisiae genome sequence for specificity confidence. qPCR assays were performed in a CFX96 real-time PCR system (Bio-Rad). Each sample was tested in duplicate in $10 \mu \mathrm{L}$ reaction mixes consisting of $5 \mu \mathrm{L}$ of SsoFast Evagreen Supermix (Bio-Rad), $0.6 \mu \mathrm{L}$ of each primer (600 nM final concentration), $2.8 \mu \mathrm{L}$ of $\mathrm{H}_{2} \mathrm{O}$, and $1 \mu \mathrm{L}$ of a $1: 10$ dilution of the 
cDNA preparation. The absence of genomic DNA in RNA samples was checked by qPCR (minus RT control). A blank (no template control) was also incorporated in each assay. The thermocycling program consisted of an initial enzyme activation step at $95^{\circ} \mathrm{C}$ during $30 \mathrm{~s}$, followed by 40 cycles of $5 \mathrm{~s}$ denaturation at $95^{\circ} \mathrm{C}$ and $5 \mathrm{~s}$ annealing/extension at $53.5^{\circ} \mathrm{C}$ (ZWF1, RPB4 and ACT1) or $55.7^{\circ} \mathrm{C}$ (PRS3 and ACT1). After completion of these cycles, data from the melting-curve were then collected to verify PCR specificity, lack of contamination and the absence of primer dimers. Relative expression levels were determined by the $2^{-\Delta \Delta \mathrm{Ct}}$ method (Livak and Schmittgen, 2001). For standardization, the results were expressed as target/reference ratio, being the reference gene the genome-encoded actin gene ( $A C T 1$ ).

\subsection{Analytical methods}

The concentrations of glucose, ethanol, acetic acid, furfural and HMF in the hydrolysates and in the samples from the fermentation runs were determined by HPLC using a Varian MetaCarb 87H column, eluted at $60^{\circ} \mathrm{C}$ with $0.005 \mathrm{M}$ sulfuric acid and at a flow rate of $0.7 \mathrm{~mL} / \mathrm{min}$. The peaks corresponding to glucose, ethanol and acetic acid were detected using a refractive index detector, whereas furfural and HMF were detected using an UV detector set at $210 \mathrm{~nm}$.

\subsection{Statistical analyses}

GraphPad Prism for Windows version 6.01 was used to carry out the statistical analyses. Differences among gene expression for CCUG53310 and PE-2 strains were determined using multiple $t$-test. Differences between the fermentation profiles of each overexpressing strain and the control strain were tested by repeated measures two-way ANOVA, followed by Bonferroni post hoc test. Differences in kinetic parameters were determined using multiple $t$-test. Statistical significance was established at $P<0.05$ for the comparisons.

\section{Results and discussion}

3.1. Differential expression of PRS3, RPB4 and ZWF1 during the initial phases of CCUG53310 and PE-2 fermentations in the presence and absence of lignocellulose-derived inhibitors

Strains adapted to tolerate superior concentrations of lignocellulose-derived inhibitors have been shown to display enhanced background expression of several genes involved in the detoxification of furfural and HMF (namely ZWF1), comparing to non-tolerant strains (Liu et al., 2009). Therefore, to better understand the role of PRS3, RPB4 and ZWF1 in the adaptation of the robust $S$. cerevisiae CCUG53310 and PE-2 strains to lignocellulose-derived inhibitors, we investigated the effect of the simultaneous presence of acetic acid, furfural and HMF (in concentrations mimicking the composition of EGW hydrolysates) on their expression at different fermentation phases (Fig. 1).

By analysing the $\mathrm{CO}_{2}$ production profiles of these strains in inhibitory and non-inhibitory fermentations (Fig. 1A), longer lag phases were necessary for the adaptation of both strains to the presence of acetic acid, furfural and HMF, as expected (Pereira et al., 2014a). The $\mathrm{CO}_{2}$ production profile of CCUG53310 was clearly more affected by inhibitory conditions than that of PE-2, reflecting the superior capacity demonstrated by the later to detoxify furfural and HMF (Fig. 1B). Indeed, PE-2 was able to completely degrade these compounds until the early exponential phase (EP), while CCUG53310 did not (Fig. 1B). When characterizing the fermentation performance of these strains in real EGW hydrolysate,
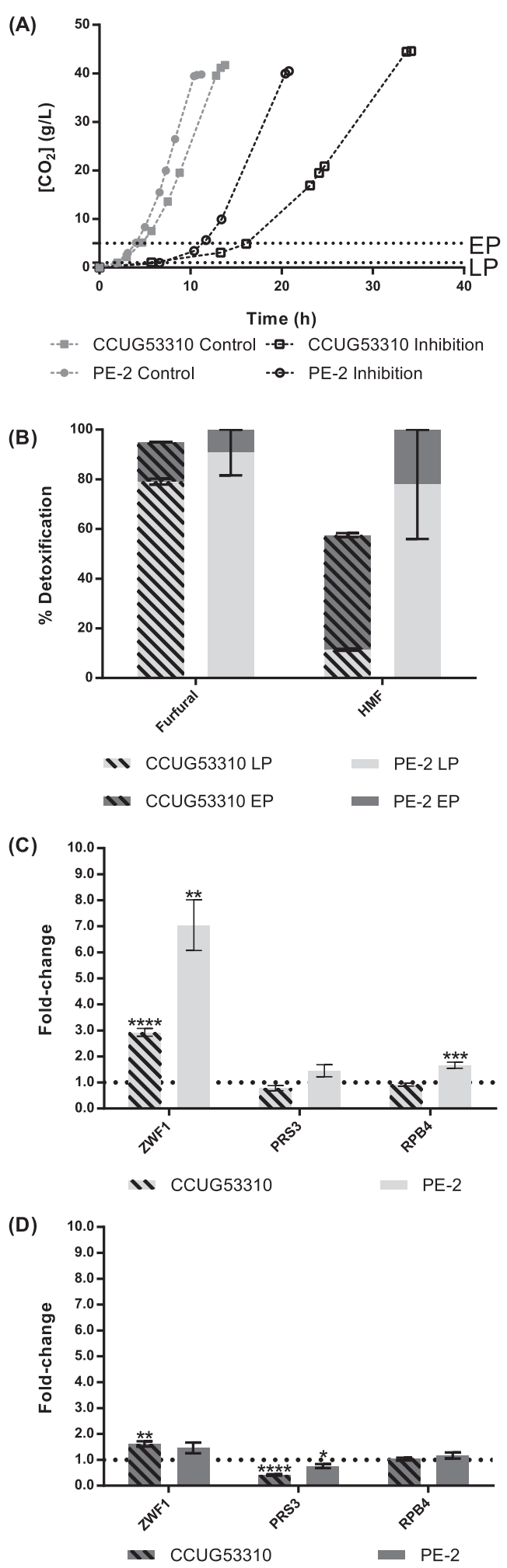

Fig. 1. Gene expression analyses of $S$. cerevisiae CCUG53310 and PE-2 during fermentations in the presence and absence of lignocellulose-derived inhibitors. (A) Profiles of $\mathrm{CO}_{2}$ production from fermentations in YPD (Control) and in YPD supplemented with acetic acid (ca. $3.1 \mathrm{~g} / \mathrm{L}$ ), HMF (ca. $0.33 \mathrm{~g} / \mathrm{L}$ ) and furfural (ca.1.7 g/ L) (Inhibition). Samples were collected at the late lag phase (LP) and early exponential phase (EP), corresponding to ca. 1 and $5 \mathrm{~g} / \mathrm{L}$ of $\mathrm{CO}_{2}$ produced respectively (indicated by the dotted lines). (B) Furfural and HMF detoxification at LP and EP of fermentation in inhibitory media. (C, D) Differential expression of $P R S 3, R P B 4$ and $Z W F 1$ at the $\mathrm{LP}(\mathrm{C})$ and $\mathrm{EP}(\mathrm{D})$ in inhibitory fermentations relative to the control (represented by the dotted line). Data represents the average \pm SEM from two biological replicates. ${ }^{*} P<0.05 ;{ }^{* *} P<0.01 ;{ }^{* * *} P<0.001 ;{ }^{* * * * *} P<0.0001$. 


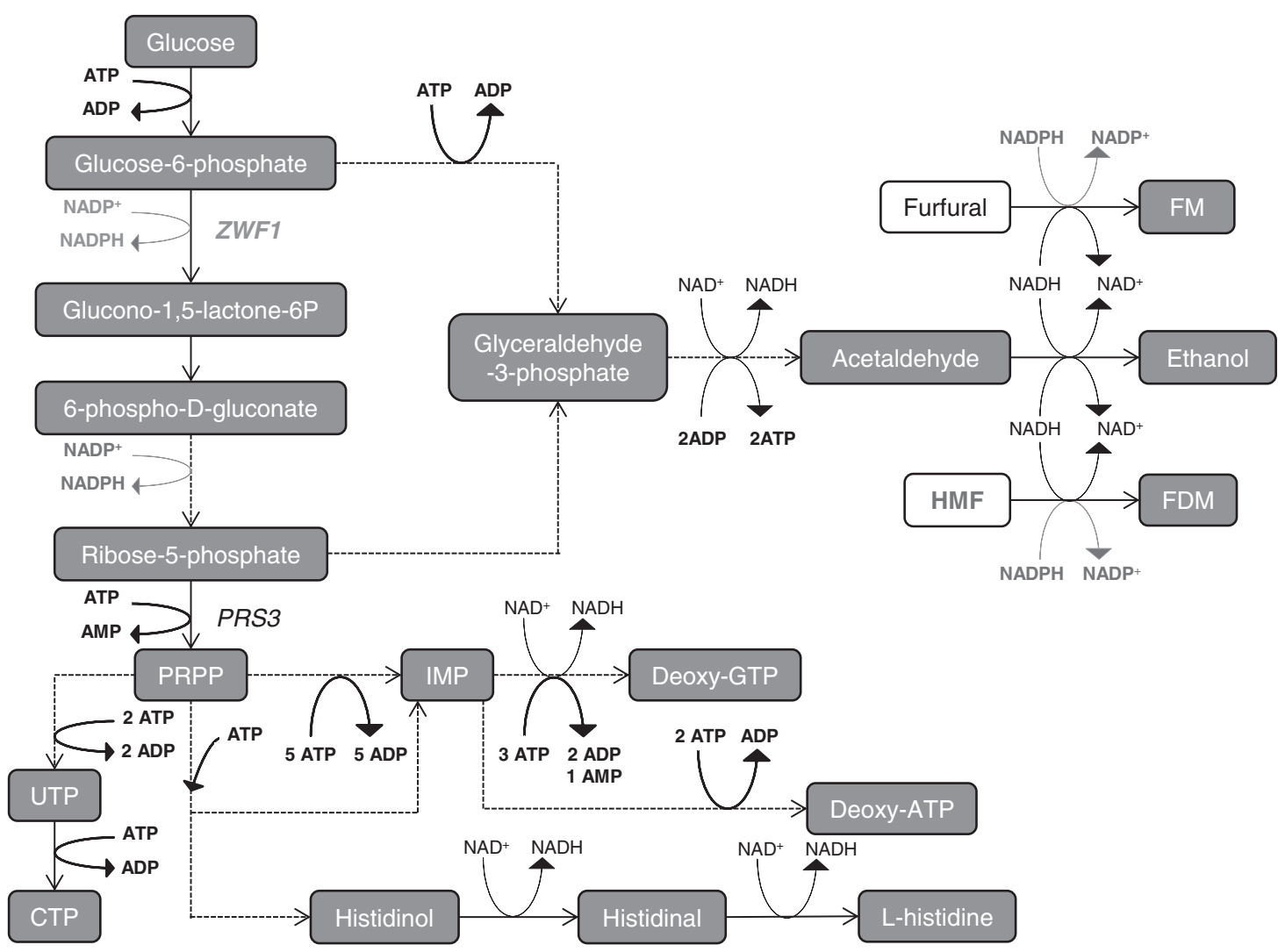

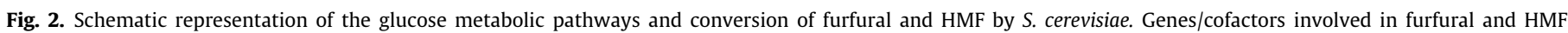

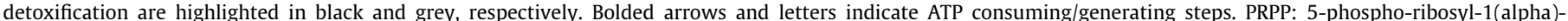

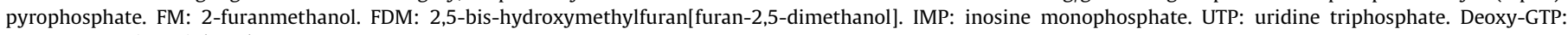
Deoxy-guanosine triphosphate.

Pereira et al. (2014a) previously reported a faster furfural detoxification rate of $S$. cerevisiae PE-2 comparatively to CCUG53310, which is in accordance with our observations.

Under anaerobic conditions, S. cerevisiae can convert furfural and HMF into less toxic corresponding alcohols (Fig. 2). Reduction of furfural is preferentially NADH-dependent, while reduction of HMF has been mainly associated with the consumption of NADPH (Wahlbom and Hahn-Hagerdal, 2002). These reductions provide sufficient $\mathrm{NAD}(\mathrm{P})^{+}$for $\mathrm{NAD}(\mathrm{P}) \mathrm{H}$ regeneration, maintaining a redox balance during the detoxification of these compounds (Liu et al., 2009). Overexpression of ZWF1 has been shown to be important for the detoxification of furfural and HMF during the lag phase (Gorsich et al., 2006; Liu et al., 2009), probably by increasing the glucose metabolism flux in favour of the pentose phosphate pathway, and thus contributing to accelerate the $\mathrm{NAD}(\mathrm{P}) \mathrm{H}$ regeneration required to supply the cofactors needed for reduction of these inhibitors (Liu et al., 2009) (Fig. 2). Accordingly, at the late lag phase (LP), ZWF1 expression was highly enhanced under inhibitory conditions in both CCUG53310 and PE-2 (Fig. 1C). At the early exponential phase (EP) (Fig. 1D), when HMF and furfural had been completely degraded by PE-2 (Fig. 1B), the expression of $Z W F 1$ in this strain reversed to levels similar to those of the control. In contrast, at this phase, ZWF1 remained slightly up-regulated in the CCUG53310 strain (Fig. 1D), probably reflecting the still incomplete furfural and HMF detoxification (Fig. 1B).

PRS3 expression at the LP (Fig. 1C) was unaffected by the simultaneous presence of acetic acid, furfural and HMF, whereas at the EP, when mainly acetic acid (PE-2) or HMF and acetic acid (CCUG53310) were present (Fig. 1B), this gene was down-regulated (Fig. 1D). In fact, the presence of acetic acid, and corresponding inhibitory effect, was permanent during the inhibitory fermentations, with the acetic acid concentration varying in the range of 1.9-3.1 g/L (average content of $2.5 \mathrm{~g} / \mathrm{L}$ ). Two mechanisms have been proposed to explain the inhibitory effect of weak acids (Russell, 1992): intracellular anion accumulation and depletion of ATP (uncoupling theory). The first proposes that the accumulation of the dissociated form of the acid inside the cell (due to the low extracellular $\mathrm{pH}$ ) leads to an intracellular acidification and consequent toxic effects at various levels of the cellular metabolism (Russell, 1992). The uncoupling theory states that the cytoplasmic $\mathrm{pH}$ decrease resultant from the inflow of weak acids activates the ATP-dependent proton pumps to neutralize the $\mathrm{pH}$, leading to a depletion of ATP (Russell, 1992). Thus, when acetic acid was the main inhibitor present (EP), the PRS3 expression was possibly down-regulated to save ATP, as Prs $3 p$ synthesizes 5 -phospho-ribosyl-1(alpha)-pyrophosphate (PRPP) in an ATPdependent reaction and PRPP is in turn a precursor of nucleotide and histidine biosynthesis, pathways with high ATP consumption (Fig. 2). At the LP this possible down-regulating effect of acetic acid was likely counteracted by the necessity of Prs $3 p$ to maintain a redox balance for furfural and HMF detoxification, as purine nucleotide and histidine biosynthesis are also important pathways for NADH regeneration (Fig. 2). Interestingly, as demonstrated by comparative transcription dynamic analyses, most of the differentially expressed genes under inhibitor challenging conditions show repressed response (Liu et al., 2009; Ma and Liu, 2010). In fact, down-regulated expression under these conditions has been suggested to provide efficient means of energy utilization for economic pathway development (Ma and Liu, 2010). 

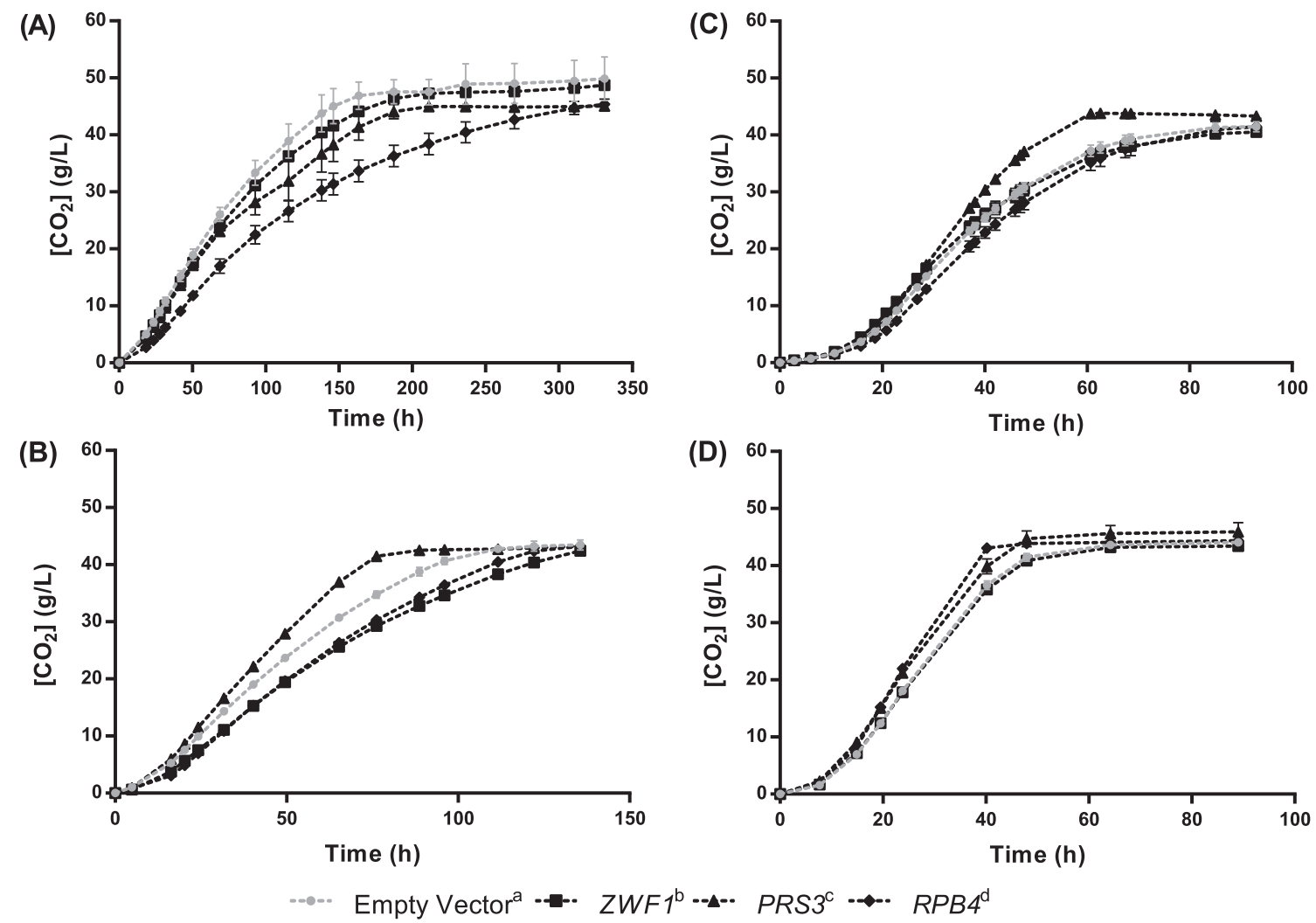

Fig. 3. $\mathrm{CO}_{2}$ production profiles of S. cerevisiae CCUG53310 transformants (Empty Vector, and overexpressing PRS3, RPB4 and ZWF1) in synthetic and real lignocellulosic hydrolysates with different inhibitors composition. Data represents the average \pm range from two biological replicates. (A) EGW hydrolysate. a,d* (from 68.7 to $211.2 \mathrm{~h}$ ). (B) Corn cob hydrolysate. a,b* (from 45.1 to 87.8 h). (C) Synthetic hydrolysate with acetic acid, HMF and furfural. a,c*. (D) Synthetic hydrolysate with furfural. a, d*. ${ }^{*} P<0.05$.

Regarding the expression of RPB4, only at the LP of PE-2 fermentations was it altered by the simultaneous presence of acetic acid, furfural and HMF, where the synergistic effect of these inhibitors induced its expression by 1.7 -fold (Fig. 1C). On the other hand, at the EP of the same fermentation, when only acetic acid was present, the expression of RPB4 was unaltered (Fig. 1D). In fact, furfural and HMF were found to repress translation initiation in $S$. cerevisiae (Iwaki et al., 2013), a process that has been shown to be stimulated by Rpb4p/Rpb7p (Harel-Sharvit et al., 2010). Rpb4p also plays an important role in the transcription of genes involved in some stress responses (Sampath and Sadhale, 2005). Together, this may support the necessity for an up-regulated expression of $R P B 4$ at an early fermentation stage, when furfural and HMF were present. However, in the CCUG53310 strain the RPB4 expression was unaffected by the presence of these stressors, which shows that different strains respond differently to the stress imposed by the toxic compounds present in lignocellulosic hydrolysates.

Consistent with the fact that PRS3, RPB4 and ZWF1 play important, but different, roles in yeast tolerance to the multiple stresses present in lignocellulosic biomass fermentation (Pereira et al., 2011, 2014b), their expression was here shown to be differentially susceptible to the presence of acetic acid, furfural and HMF (Fig. 1C and D). The different inhibitor concentrations at different fermentation stages were also shown to elicit distinct gene expression responses (Fig. 1B-D), which also varied between the two strains studied. S. cerevisiae PE-2, which displayed a faster adaptation to the inhibitory conditions here tested (Fig. 1A), as well as a more efficient furfural and HMF detoxification capacity than $S$. cerevisiae CCUG53310 (Fig. 1B), also presented a more highly induced expression of ZWF1 and RPB4 in the presence of acetic acid, furfural and HMF than the later (Fig. 1C), highlighting the importance of these genes for maximal yeast tolerance to these inhibitors.
3.2. Effect of PRS3, RPB4 and ZWF1 overexpression on the fermentation performance of S. cerevisiae CCUG53310 in lignocellulosic hydrolysates

Since the strain background and the hydrolysate inhibitory load have been shown to differentially influence the outcome of genetic manipulations targeting the improvement of strain resistance to lignocellulose-derived inhibitors (Alriksson et al., 2010; Park et al., 2011; Wallace-Salinas et al., 2014), we here assessed the effect of PRS3, RPB4 and ZWF1 overexpression on the fermentation performance of two already robust background strains under process-like conditions.

The $\mathrm{CO}_{2}$ production profiles of $S$. cerevisiae CCUG53310 independently overexpressing $P R S 3, R P B 4$ or ZWF1 were compared with the control fermentation profiles in different real and synthetic hydrolysates (Fig. 3). For each hydrolysate, the lag phase, the maximum fermentation rate, the maximum $\mathrm{CO}_{2}$ productivity and the final ethanol concentration of each transformant are indicated in Table 2.

PRS3 overexpression improved the S. cerevisiae CCUG53310 fermentation performance in all the hydrolysates tested, with the exception of EGW hydrolysate (Fig. 3, Table 2), where no significant differences where observed between the fermentative profiles of the PRS3-overexpressing strain and the control strain (Fig. 3A, Table 2). However, in the synthetic hydrolysate containing acetic acid, furfural and HMF in concentrations similar to those of the EGW hydrolysate (Fig. 3C), the overexpression of this gene led to a $20 \%$ faster fermentation rate and to a $48 \%$ higher $\mathrm{CO}_{2}$ productivity than the control (Table 2). Similarly, PRS3 overexpression also led to improved fermentation rate $(32 \%)$ and $\mathrm{CO}_{2}$ productivity $(42 \%)$ in corn cob hydrolysate (Fig. 3B, Table 2). The total inhibitory load of the corn cob hydrolysate is lower than that of the EGW hydrolysate, particularly in what concerns acetic acid concentration 
Table 2

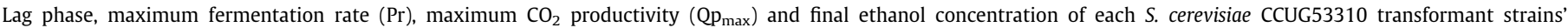

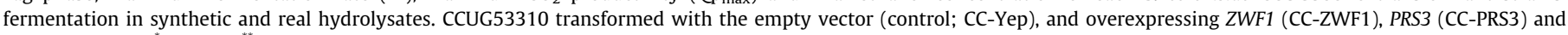
RPB4 (CC-RPB4). ${ }^{*} P<0.05 ;{ }^{* *} P<0.01$.

\begin{tabular}{|c|c|c|c|c|}
\hline Strains & Lag phase (h) & $\operatorname{Pr}\left(h^{-1}\right)$ & $\mathrm{Qp}_{\max }(\mathrm{g} /(\mathrm{L} h))$ & Ethanol $(\mathrm{g} / \mathrm{L})$ \\
\hline \multicolumn{5}{|c|}{ EGW hydrolysate: $2.33 \mathrm{~g} / \mathrm{L}$ acetic acid, $1.77 \mathrm{~g} / \mathrm{L}$ furfural, $0.26 \mathrm{~g} / \mathrm{L} H M F$ and $113 \mathrm{~g} / \mathrm{L}$ glucose } \\
\hline CC-Yep & $18.3 \pm 0.0$ & $0.0352 \pm 0.0002$ & $0.254 \pm 0.016$ & $52.0 \pm 5.6$ \\
\hline CC-ZWF1 & $18.3 \pm 0.0$ & $0.0319 \pm 0.0002^{* *}$ & $0.224 \pm 0.004$ & $50.7 \pm 0.7$ \\
\hline CC-PRS3 & $18.3 \pm 0.0$ & $0.0298 \pm 0.0066$ & $0.213 \pm 0.003$ & $46.9 \pm 0.6$ \\
\hline CC-RPB4 & $18.3 \pm 0.0$ & $0.0238 \pm 0.0018^{*}$ & $0.144 \pm 0.005^{*}$ & $47.2 \pm 1.3$ \\
\hline \multicolumn{5}{|c|}{ Corn cob hydrolysate: $1.57 \mathrm{~g} / \mathrm{L}$ acetic acid, $1.54 \mathrm{~g} / \mathrm{L}$ furfural, $0.12 \mathrm{~g} / \mathrm{L} \mathrm{HMF}$ and $112 \mathrm{~g} / \mathrm{L}$ glucose } \\
\hline CC-YEp & $16.3 \pm 0.0$ & $0.0570 \pm 0.0048$ & $0.384 \pm 0.006$ & $45.2 \pm 1.3$ \\
\hline CC-ZWF1 & $16.3 \pm 0.0$ & $0.0484 \pm 0.0005$ & $0.313 \pm 0.005^{* *}$ & $44.1 \pm 0.7$ \\
\hline CC-PRS3 & $16.3 \pm 0.0$ & $0.0750 \pm 0.0010^{*}$ & $0.544 \pm 0.007^{* *}$ & $45.0 \pm 0.4$ \\
\hline CC-RPB4 & $16.3 \pm 0.0$ & $0.0505 \pm 0.0014$ & $0.347 \pm 0.005^{*}$ & $45.0 \pm 0.8$ \\
\hline \multicolumn{5}{|c|}{ Syntethic hydrolysate 1: $2.71 \mathrm{~g} / \mathrm{L}$ acetic acid, $1.28 \mathrm{~g} / \mathrm{L}$ furfural, $0.34 \mathrm{~g} / \mathrm{L} \mathrm{HMF}$ and $120 \mathrm{~g} / \mathrm{L}$ glucose } \\
\hline CC-YEp & $15.8 \pm 0.0$ & $0.102 \pm 0.000$ & $0.486 \pm 0.013$ & $43.1 \pm 1.3$ \\
\hline CC-ZWF1 & $15.8 \pm 0.0$ & $0.0995 \pm 0.0023$ & $0.474 \pm 0.009$ & $42.1 \pm 0.8$ \\
\hline CC-PRS3 & $15.8 \pm 0.0$ & $0.122 \pm 0.004^{*}$ & $0.721 \pm 0.010^{* *}$ & $45.0 \pm 0.9$ \\
\hline CC-RPB4 & $15.8 \pm 0.0$ & $0.0973 \pm 0.0005^{* *}$ & $0.481 \pm 0.024$ & $43.1 \pm 2.2$ \\
\hline \multicolumn{5}{|c|}{ Syntethic hydrolysate 2: $1.95 \mathrm{~g} / \mathrm{L}$ furfural and $112 \mathrm{~g} / \mathrm{L}$ glucose } \\
\hline CC-YEp & $7.7 \pm 0.0$ & $0.137 \pm 0.007$ & $0.680 \pm 0.001$ & $45.8 \pm 0.3$ \\
\hline CC-ZWF1 & $7.7 \pm 0.0$ & $0.133 \pm 0.002$ & $0.673 \pm 0.001^{*}$ & $45.2 \pm 0.0$ \\
\hline CC-PRS3 & $7.7 \pm 0.0$ & $0.144 \pm 0.001$ & $0.934 \pm 0.041^{*}$ & $47.8 \pm 2.3$ \\
\hline CC-RPB4 & $7.7 \pm 0.0$ & $0.179 \pm 0.004^{*}$ & $1.07 \pm 0.02^{* *}$ & $46.1 \pm 0.0$ \\
\hline \multicolumn{5}{|c|}{ Syntethic hydrolysate base: minimal medium with $120 \mathrm{~g} / \mathrm{L}$ glucose } \\
\hline CC-Yep & $3.6 \pm 0.0$ & $0.297 \pm 0.013$ & $1.95 \pm 0.01$ & $46.2 \pm 0.2$ \\
\hline CC-ZWF1 & $3.6 \pm 0.0$ & $0.286 \pm 0.005$ & $1.91 \pm 0.07$ & $45.3 \pm 1.8$ \\
\hline CC-PRS3 & $3.6 \pm 0.0$ & $0.283 \pm 0.009$ & $1.95 \pm 0.01$ & $46.1 \pm 0.3$ \\
\hline CC-RPB4 & $3.6 \pm 0.0$ & $0.284 \pm 0.001$ & $1.90 \pm 0.02$ & $45.4 \pm 0.6$ \\
\hline
\end{tabular}
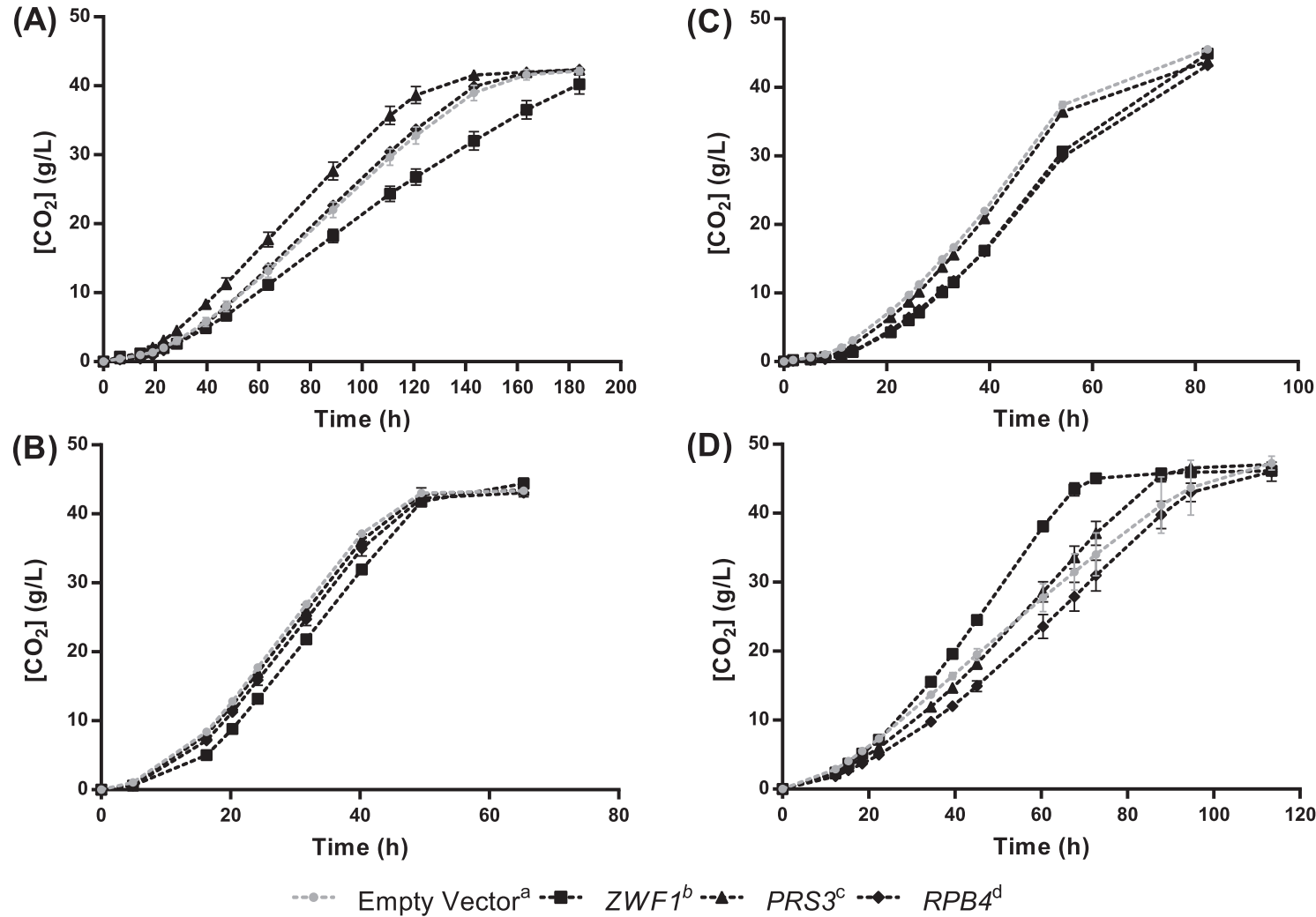

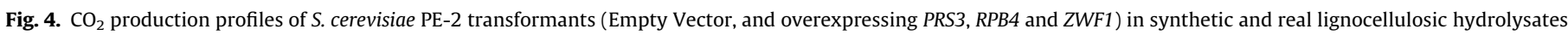

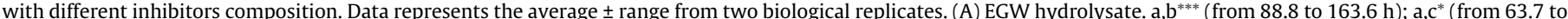

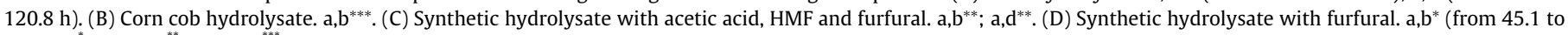
87.8 h). ${ }^{*} P<0.05 ;{ }^{* *} P<0.01 ;{ }^{* * *} P<0.001$. 
Table 3

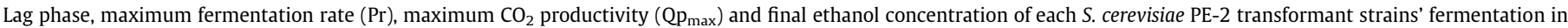

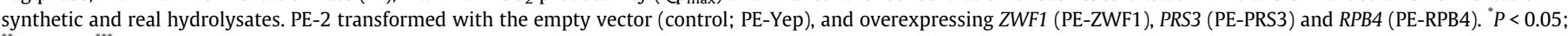
${ }^{* *} P<0.01 ;{ }^{* * *} P<0.001$.

\begin{tabular}{|c|c|c|c|c|}
\hline Strain & Lag phase $(\mathrm{h})$ & $\operatorname{Pr}\left(\mathrm{h}^{-1}\right)$ & $\mathrm{Qp}_{\max }(\mathrm{g} /(\mathrm{Lh}))$ & Ethanol $(\mathrm{g} / \mathrm{L})$ \\
\hline \multicolumn{5}{|c|}{ EGW hydrolysate: $1.80 \mathrm{~g} / \mathrm{L}$ acetic acid, $1.11 \mathrm{~g} / \mathrm{L}$ furfural, $0.24 \mathrm{~g} / \mathrm{L} \mathrm{HMF}$ and $111 \mathrm{~g} / \mathrm{L}$ glucose } \\
\hline PE-Yep & $28.4 \pm 0.0$ & $0.0400 \pm 0.0000$ & $0.254 \pm 0.007$ & $43.8 \pm 0.7$ \\
\hline PE-ZWF1 & $28.4 \pm 0.0$ & $0.0338 \pm 0.0017^{*}$ & $0.219 \pm 0.011$ & $41.8 \pm 2.0$ \\
\hline PE-PRS3 & $28.4 \pm 0.0$ & $0.0471 \pm 0.0021^{*}$ & $0.290 \pm 0.004^{*}$ & $44.1 \pm 0.1$ \\
\hline PE-RPB4 & $28.4 \pm 0.0$ & $0.0422 \pm 0.0001^{* * *}$ & $0.256 \pm 0.000$ & $44.0 \pm 0.3$ \\
\hline \multicolumn{5}{|c|}{ Corn cob hydrolysate: $1.57 \mathrm{~g} / \mathrm{L}$ acetic acid, $1.54 \mathrm{~g} / \mathrm{L}$ furfural, $0.12 \mathrm{~g} / \mathrm{L} \mathrm{HMF}$ and $112 \mathrm{~g} / \mathrm{L}$ glucose } \\
\hline PE-YEp & $16.3 \pm 0.0$ & $0.132 \pm 0.002$ & $0.869 \pm 0.007$ & $45.1 \pm 0.4$ \\
\hline PE-ZWF1 & $16.3 \pm 0.0$ & $0.123 \pm 0.005$ & $0.844 \pm 0.003^{*}$ & $46.2 \pm 1.1$ \\
\hline PE-PRS3 & $16.3 \pm 0.0$ & $0.129 \pm 0.003$ & $0.865 \pm 0.027$ & $45.2 \pm 1.5$ \\
\hline PE-RPB4 & $16.3 \pm 0.0$ & $0.128 \pm 0.001$ & $0.854 \pm 0.018$ & $44.8 \pm 1.0$ \\
\hline \multicolumn{5}{|c|}{ Syntethic hydrolysate 1: $3.00 \mathrm{~g} / \mathrm{L}$ acetic acid, $1.46 \mathrm{~g} / \mathrm{L}$ furfural, $0.37 \mathrm{~g} / \mathrm{L} \mathrm{HMF}$ and $120 \mathrm{~g} / \mathrm{L}$ glucose } \\
\hline PE-YEp & $13.4 \pm 0.0$ & $0.0977 \pm 0.0017$ & $0.553 \pm 0.001$ & $47.4 \pm 0.1$ \\
\hline PE-ZWF1 & $20.8 \pm 0.0$ & $0.0900 \pm 0.0007^{*}$ & $0.546 \pm 0.006$ & $43.7 \pm 0.5$ \\
\hline PE-PRS3 & $13.4 \pm 0.0$ & $0.103 \pm 0.001$ & $0.532 \pm 0.005^{*}$ & $45.6 \pm 0.5^{*}$ \\
\hline PE-RPB4 & $20.8 \pm 0.0$ & $0.0893 \pm 0.0001^{*}$ & $0.524 \pm 0.001^{* *}$ & $44.9 \pm 0.1^{* *}$ \\
\hline \multicolumn{5}{|c|}{ Syntethic hydrolysate $2: 2.52 \mathrm{~g} / \mathrm{L}$ furfural and $106 \mathrm{~g} / \mathrm{L}$ glucose } \\
\hline PE-YEp & $12.3 \pm 0.0$ & $0.0535 \pm 0.0050$ & $0.417 \pm 0.009$ & $49.2 \pm 1.0$ \\
\hline PE-ZWF1 & $12.3 \pm 0.0$ & $0.0895 \pm 0.0010^{* *}$ & $0.620 \pm 0.005^{* *}$ & $48.0 \pm 0.3$ \\
\hline PE-PRS3 & $12.3 \pm 0.0$ & $0.0632 \pm 0.0034$ & $0.518 \pm 0.011^{* *}$ & $48.9 \pm 0.3$ \\
\hline PE-RPB4 & $15.3 \pm 0.0$ & $0.0555 \pm 0.0057$ & $0.406 \pm 0.012$ & $47.9 \pm 1.4$ \\
\hline \multicolumn{5}{|c|}{ Syntethic hydrolysate base: minimal medium with $120 \mathrm{~g} / \mathrm{L}$ glucose } \\
\hline PE-YEp & $6.5 \pm 0.0$ & $0.168 \pm 0.003$ & $1.12 \pm 0.01$ & $45.4 \pm 0.4$ \\
\hline PE-ZWF1 & $6.5 \pm 0.0$ & $0.176 \pm 0.002$ & $1.15 \pm 0.01$ & $47.1 \pm 0.4^{*}$ \\
\hline PE-PRS3 & $6.5 \pm 0.0$ & $0.169 \pm 0.008$ & $1.13 \pm 0.05$ & $45.8 \pm 2.5$ \\
\hline PE-RPB4 & $6.5 \pm 0.0$ & $0.170 \pm 0.002$ & $1.13 \pm 0.01$ & $45.8 \pm 0.4$ \\
\hline
\end{tabular}

(Table 2). As mentioned above, PRS3 overexpression may contribute to increase the carbon flux in favour of metabolic pathways important for the regeneration of $\mathrm{NADH}$, a cofactor required for the detoxification of furfural and HMF, and for ethanol production (Fig. 2). However, some of these pathways compete for ATP with cellular mechanisms that are activated to counteract the cytoplasmic acidification promoted by the inflow of acetic acid (Russell, 1992). Therefore, in the presence of relatively high concentrations of acetic acid, PRS3 overexpression may not be particularly advantageous.

The overexpression of RPB4 had contrasting effects over the fermentation performance of $S$. cerevisiae CCUG53310 in different inhibitory media (Fig. 3). Its overexpression was advantageous for the fermentation in synthetic hydrolysate containing only furfural (Fig. 3D), allowing a $57 \%$ higher $\mathrm{CO}_{2}$ productivity and a $31 \%$ faster fermentation rate than the control. However, RPB4 overexpression negatively affected the fermentation performance of $S$. cerevisiae CCUG53310 in more complex hydrolysates (Table 2). In the synthetic hydrolysate containing acetic acid, furfural and HMF (Fig. 3C), the overexpression of RPB4 slowed down the CCUG53310 fermentation rate (Table 2). RPB4 overexpression also reduced the CCUG53310 fermentation rate in EGW hydrolysate by $48 \%$. Furthermore, the $\mathrm{CO}_{2}$ productivity in corn cob and EGW hydrolysate fermentations was also negatively affected by the overexpression of RPB4 (Table 2). Therefore, although RPB4 overexpression was advantageous for the fermentation performance of $S$. cerevisiae CCUG53310 at relatively high initial furfural concentrations, under more complex inhibitory conditions it had a deleterious effect over this strain physiology.

ZWF1 overexpression in laboratorial $S$. cerevisiae strains was previously described to allow growth in the presence of otherwise lethal concentrations of furfural (Gorsich et al., 2006) and to improve furfural and HMF conversion rates, with consequent increase in ethanol production and cell growth (Park et al., 2011). Nevertheless, ZWF1 overexpression was here shown to negatively affect the fermentation performance of the $S$. cerevisiae
CCUG53310 strain in real hydrolysates (Fig. 3A and B, Table 2). It is worth noting that this gene is already naturally overexpressed by CCUG53310 upon exposure to lignocellulosic inhibitors (Figs. 1C and D), and our results show that its additional overexpression brings no extra advantage.

\subsection{Effect of PRS3, RPB4 and ZWF1 overexpression on the fermentation performance of $S$. cerevisiae PE-2 in lignocellulosic hydrolysates}

The $\mathrm{CO}_{2}$ production profiles of $S$. cerevisiae $\mathrm{PE}-2$ independently overexpressing PRS3, RPB4 or ZWF1 were also compared with control fermentation profiles in different real and synthetic hydrolysates (Fig. 4). For each hydrolysate, the lag phase, the maximum fermentation rate, the maximum $\mathrm{CO}_{2}$ productivity and the final ethanol concentration of each transformant are indicated in Table 3 .

As seen in Fig. 4A and Table 3, the overexpression of PRS3 improved the $S$. cerevisiae PE-2 fermentation rate (18\%) and $\mathrm{CO}_{2}$ productivity (14\%) in EGW hydrolysate. A $24 \%$ increase on the $\mathrm{CO}_{2}$ productivity of the PE-2 PRS3-overexpressing strain was also observed in synthetic hydrolysate containing only furfural at a relatively high concentration (Fig. 4D, Table 3). Similarly, the PE-2 $\mathrm{CO}_{2}$ productivity in this hydrolysate was also significantly improved (49\%) by the overexpression of $Z W F 1$, as was its fermentation rate (Fig. 4D, Table 3). However, ZWF1 overexpression had a negative effect over the $S$. cerevisiae PE-2 fermentation performance in all the other hydrolysates tested (Fig. 4, Table 3). Depending on the inhibitory composition of the hydrolysate, the overexpression of RPB4 also had contrasting outcomes in the fermentation performance of PE-2. While in synthetic hydrolysates its overexpression increased the lag-phase, in EGW hydrolysate it slightly improved the PE-2 fermentation rate (Table 3 ).

Similarly to what was observed in the S. cerevisiae CCUG53310 strain, ZWF1 and RPB4 overexpression in PE-2 also resulted mainly in negative or no effects over its fermentation performance. On the other hand, PRS3 overexpression produced more consistent and 
advantageous effects over the fermentation capacities of these strains.

The overexpression of $P R S 3, R P B 4$ and $Z W F 1$ was here shown to present different outcomes that were clearly dependent on the inhibitory composition of the fermentation media, but also on the background strain. For instance, in the hydrolysate containing only furfural, the overexpression of RPB4 had a positive effect in CCUG53310 (Fig. 3D), but a negative effect in the PE-2 strain (Fig. 4D). Since Rpb4p has been suggested to be involved in the regulation of the cellular response to certain stress conditions (Sampath and Sadhale, 2005), our results hint at the existence of regulatory differences between these strains. In fact, an alignment of the promoter region of $P R S 3, R P B 4$ and ZWF1 from several laboratory and industrial background S. cerevisiae strains (S288, SIGMA1278b, CEN.PK113-7D, AWRI1631, EC1118, CBS7960 and JAY291, haploid derivative of PE-2) revealed relevant differences between strains, namely in terms of TATA box location and transcription factors' binding sites. Moreover, slight differences in the amino acid sequence of Zwf1p were also found between these strains and Moon and Liu (2012) showed that even slight alterations in the coding sequence of another gene involved in furfural and HMF detoxification, GRE2, resulted in increased growth rates in the presence of HMF. Although the CCUG53310 genome sequence is not publically available, similar differences may also exist between this strain and PE-2, which may help explain the different responses of CCUG53310 and PE-2 to similar fermentation conditions and to the overexpression of PRS3, RPB4 and ZWF1.

Considering the results presented here, it is clear that the engineering of yeast strains for increased tolerance to inhibitory lignocellulosic biomass must be carefully addressed, considering the different background of each $S$. cerevisiae strain and the specific composition of the raw material and hydrolysate to use.

\section{Conclusions}

S. cerevisiae CCUG53310 and PE-2 were here shown to differentially express $P R S 3, R P B 4$ and $Z W F 1$ in response to different lignocellulosic inhibitor loads. Increased expression of ZWF1 and RPB4 in the presence of acetic acid, furfural and HMF was found to contribute to yeast adaptation to these inhibitors, but their overexpression did not improve the fermentation performance in real lignocellulosic hydrolysates. In contrast, PRS3 overexpression improved the fermentation rate and productivity of both strains. The heterogeneous outcomes of these genes overexpression in different hydrolysates show that tolerance engineering must be customized to the strain background and hydrolysate used in the process.

\section{Acknowledgements}

This work was financially supported by Fundação para a Ciência e a Tecnologia, Portugal, through: Grant SFRH/BDP/63831/2009 to C. Oliveira; grant SFRH/BPD/77995/2011 to A. Romaní; Strategic Project PEst-OE/EQB/LA0023/2013; and Project BioInd NORTE-07-0124-FEDER-000028 co-funded by the Programa Operacional Regional do Norte (ON.2 - O Novo Norte), QREN, FEDER.

\section{Appendix A. Supplementary data}

Supplementary data associated with this article can be found, in the online version, at http://dx.doi.org/10.1016/j.biortech.2015.05. 006.

\section{References}

Alriksson, B., Horvath, I.S., Jonsson, L.J., 2010. Overexpression of Saccharomyces cerevisiae transcription factor and multidrug resistance genes conveys enhanced resistance to lignocellulose-derived fermentation inhibitors. Process Biochem. 45, 264-271.

Bajwa, P.K., Ho, C.Y., Chan, C.K., Martin, V.J.J., Trevors, J.T., Lee, H., 2013. Transcriptional profiling of Saccharomyces cerevisiae T2 cells upon exposure to hardwood spent sulphite liquor: comparison to acetic acid, furfural and hydroxymethylfurfural. Antonie van Leeuwenhoek 103, 1281-1295.

Basso, L.C., de Amorim, H.V., de Oliveira, A.J., Lopes, M.L., 2008. Yeast selection for fuel ethanol production in Brazil. FEMS Yeast Res. 8, 1155-1163.

Chandel, A.K., da Silva, S.S., Singh, O.V., 2013. Detoxification of lignocellulose hydrolysates: biochemical and metabolic engineering toward white biotechnology. Bioenerg. Res. 6, 388-401.

Gietz, R.D., Sugino, A., 1988. New yeast-Escherichia coli shuttle vectors constructed with in vitro mutagenized yeast genes lacking six-base pair restriction sites. Gene 74, 527-534.

Gietz, D., St Jean, A., Woods, R.A., Schiestl, R.H., 1992. Improved method for high efficiency transformation of intact yeast cells. Nucleic Acids Res. 20, 1425.

Gorsich, S.W., Dien, B.S., Nichols, N.N., Slininger, P.J., Liu, Z.L., Skory, C.D., 2006 Tolerance to furfural-induced stress is associated with pentose phosphate pathway genes ZWF1, GND1, RPE1, and TKL1 in Saccharomyces cerevisiae. Appl. Microbiol. Biotechnol. 71, 339-349.

Guldener, U., Heck, S., Fiedler, T., Beinhauer, J., Hegemann, J.H., 1996. A new efficient gene disruption cassette for repeated use in budding yeast. Nucleic Acids Res. 24, 2519-2524.

Harel-Sharvit, L., Eldad, N., Haimovich, G., Barkai, O., Duek, L., Choder, M., 2010. RNA Polymerase II subunits link transcription and mRNA decay to translation. Cell $143,552-563$.

Iwaki, A. Kawai, T, Yamamoto, Y. Izawa, S., 2013. Biomass conversion inhibitors furfural and 5-hydroxymethylfurfural induce formation of messenger RNP granules and attenuate translation activity in Saccharomyces cerevisiae. Appl, Environ. Microbiol. 79, 1661-1667.

Lavoie, J.M., Capek-Menard, E., Gauvin, H., Chornet, E., 2010. Production of pulp from Salix viminalis energy crops using the FIRSST process. Bioresour. Technol. 101, 4940-4946.

Liu, Z.L., Ma, M., Song, M., 2009. Evolutionarily engineered ethanologenic yeast detoxifies lignocellulosic biomass conversion inhibitors by reprogrammed pathways. Mol. Genet. Genomics 282, 233-244.

Livak, K.J., Schmittgen, T.D., 2001. Analysis of relative gene expression data using real-time quantitative PCR and the $2^{-\Delta \Delta \mathrm{Ct}}$ method. Methods $25,402-408$.

Ma, M.G., Liu, Z.L., 2010. Comparative transcriptome profiling analyses during the lag phase uncover YAP1, PDR1, PDR3, RPN4, and HSF1 as key regulatory genes in genomic adaptation to the lignocellulose derived inhibitor HMF for Saccharomyces cerevisiae. BMC Genomics 11, 660.

Mira, N.P., Teixeira, M.C., Sa-Correia, I., 2010. Adaptive response and tolerance to weak acids in Saccharomyces cerevisiae: a genome-wide view. OMICS 14, 525540.

Modig, T., Almeida, J.R.M., Gorwa-Grauslund, M.F., Liden, G., 2008. Variability of the response of Saccharomyces cerevisiae strains to lignocellulose hydrolysate. Biotechnol. Bioeng. 100, 423-429.

Moon, J., Liu, Z.L., 2012. Engineered NADH-dependent GRE2 from Saccharomyces cerevisiae by directed enzyme evolution enhances HMF reduction using additional cofactor NADPH. Enzyme Microb. Technol. 50, 115-120.

Parawira, W., Tekere, M., 2011. Biotechnological strategies to overcome inhibitors in lignocellulose hydrolysates for ethanol production: review. Crit. Rev. Biotechnol. 31, 20-31.

Park, S.E, Koo, H.M. Park, Y.K. Park, S.M. Park, J.C. Lee, O.K. Park, Y.C., Seo, J.H., 2011. Expression of aldehyde dehydrogenase 6 reduces inhibitory effect of furan derivatives on cell growth and ethanol production in Saccharomyces cerevisiae. Bioresour. Technol. 102, 6033-6038.

Pereira, F.B., Guimaraes, P.M., Gomes, D.G., Mira, N.P., Teixeira, M.C., Sa-Correia, I., Domingues, L., 2011. Identification of candidate genes for yeast engineering to improve bioethanol production in very high gravity and lignocellulosic biomass industrial fermentations. Biotechnol. Biofuels 4, 57.

Pereira, F.B., Romani, A., Ruiz, H.A., Teixeira, J.A., Domingues, L., 2014a. Industrial robust yeast isolates with great potential for fermentation of lignocellulosic biomass. Bioresour. Technol. 161, 192-199.

Pereira, F.B., Teixeira, M.C., Mira, N.P., Sa-Correia, I., Domingues, L., 2014b. Genomewide screening of Saccharomyces cerevisiae genes required to foster tolerance towards industrial wheat straw hydrolysates. J. Ind. Microbiol. Biotechnol. 41, 1753-1761.

Petersson, A., Almeida, J.R., Modig, T., Karhumaa, K., Hahn-Hagerdal, B., GorwaGrauslund, M.F., Liden, G., 2006. A 5-hydroxymethyl furfural reducing enzyme encoded by the Saccharomyces cerevisiae ADH6 gene conveys HMF tolerance. Yeast 23, 455-464.

Purwadi, R., Brandberg, T., Taherzadeh, M.J., 2007. A possible industrial solution to ferment lignocellulosic hydrolyzate to ethanol: continuous cultivation with flocculating yeast. Int. J. Mol. Sci. 8, 920-932.

Rodrigues, L., Moldes, A., Teixeira, J., Oliveira, R., 2006. Kinetic study of fermentative biosurfactant production by Lactobacillus strains. Biochem. Eng. J. 28, 109-116.

Romani, A., Pereira, F., Johansson, B., Domingues, L., 2015. Metabolic engineering of Saccharomyces cerevisiae ethanol strains PE-2 and CAT-1 for efficient lignocellulosic fermentation. Bioresour. Technol. 179, 150-158. 
Russell, J.B., 1992. Another explanation for the toxicity of fermentation acids at low ph - anion accumulation versus uncoupling. J. Appl. Bacteriol. 73, 363-370.

Sampath, V., Sadhale, P., 2005. Rpb4 and Rpb7: a sub-complex integral to multisubunit RNA polymerases performs a multitude of functions. IUBMB Life 57, 93-102.

Wahlbom, C.F., Hahn-Hagerdal, B., 2002. Furfural, 5-hydroxymethyl furfural, and acetoin act as external electron acceptors during anaerobic fermentation of xylose in recombinant Saccharomyces cerevisiae. Biotechnol. Bioeng. 78, 172178.
Wallace-Salinas, V., Signori, L., Li, Y.Y., Ask, M., Bettiga, M., Porro, D., Thevelein, J.M. Branduardi, P., Foulquie-Moreno, M.R., Gorwa-Grauslund, M., 2014. Reassessment of YAP1 and MCR1 contributions to inhibitor tolerance in robust engineered Saccharomyces cerevisiae fermenting undetoxified lignocellulosic hydrolysate. AMB Express 4, 56.

Westman, J.O., Taherzadeh, M.J., Franzen, C.J., 2012. Inhibitor tolerance and flocculation of a yeast strain suitable for second generation bioethanol production. Electron. J. Biotechnol. 15. 